\title{
The equality of generalized matrix functions on the set of all symmetric matrices
}

\author{
Ratsiri Sanguanwong ${ }^{\mathrm{a}}$, Kijti Rodtes ${ }^{\mathrm{b}, *}$ \\ ${ }^{a}$ Department of Mathematics, Faculty of Science, Naresuan University, Phitsanulok \\ 65000, Thailand \\ ${ }^{b}$ Department of Mathematics, Faculty of Science, Naresuan University, and Research \\ Center for Academic Excellent in Mathematics, Phitsanulok 65000, Thailand
}

\begin{abstract}
A generalized matrix function $d_{\chi}^{G}: M_{n}(\mathbb{C}) \rightarrow \mathbb{C}$ is a function constructed by a subgroup $G$ of $S_{n}$ and a complex valued function $\chi$ of $G$. The main purpose of this paper is to find a necessary and sufficient condition for the equality of two generalized matrix functions on the set of all symmetric matrices, $\mathbb{S}_{n}(\mathbb{C})$. In order to fulfill the purpose, a symmetric matrix $S_{\sigma}$ is constructed and $d_{\chi}^{G}\left(S_{\sigma}\right)$ is evaluated for each $\sigma \in S_{n}$. By applying the value of $d_{\chi}^{G}\left(S_{\sigma}\right)$, it is shown that $d_{\chi}^{G}(A B)=d_{\chi}^{G}(A) d_{\chi}^{G}(B)$ for each $A, B \in \mathbb{S}_{n}(\mathbb{C})$ if and only if $d_{\chi}^{G}=$ det. Furthermore, a criterion when $d_{\chi}^{G}(A B)=d_{\chi}^{G}(B A)$ for every $A, B \in \mathbb{S}_{n}(\mathbb{C})$, is established.
\end{abstract}

Keywords: Generalized matrix function, Symmetric matrix, Symmetric group

2010 MSC: $15 \mathrm{~A} 15$

\section{Introduction}

In the theory of matrix, a determinant and a permanent are two wellknown functions having many useful applications. A concept of a generalized matrix function is to generalize notions of permanents and determinants. Jafari and Madadi showed that two generalized matrix functions $d_{\varphi}^{H}$ and $d_{\psi}^{K}$ are equal on the set of all nonsingular matrices if and only if the extended

\footnotetext{
*Corresponding author

Email addresses: r.sanguanwong@gmail.com (Ratsiri Sanguanwong), kijtir@nu.ac.th (Kijti Rodtes)
} 
function $\hat{\varphi}$ of $\varphi$ and $\hat{\psi}$ of $\psi$ are equal. In fact, by applying the proof of their results, the condition also holds for $M_{n}(\mathbb{C}),[3]$.

In 1986, Bosch discovered the factorization of a square matrix in term of the product of two symmetric matrices, [1], which causes our attraction to characterize the equality of generalized matrix functions on the set of all symmetric matrices. If $\varphi$ is not a real valued function and $\psi$ conjugates to $\varphi$, we can calculate that $d_{\varphi}^{H}$ and $d_{\psi}^{K}$ is equal on the set of all symmetric matrices. We construct a symmetric matrix $S_{\sigma}$, for each $\sigma \in S_{n}$ and define an equivalence relation on $S_{n}$. By using such matrices along with the relation, a necessary and sufficient condition for the equality is proven (see Theorem [3.9). By applying $S_{\sigma}$ for some $\sigma \in S_{n}$, we obtain Theorem 3.17 which related to Corollary 2.4. in [2]. By these results, to obtain the equalities on the set of all symmetric matrices, it suffices to work on the permutation group which is finite.

\section{Preliminaries}

Let $G$ be a subgroup of $S_{n}$. A character of $G$ is a complex valued function of $G$ defined by $g \mapsto \operatorname{tr}\left(\varphi_{g}\right)$, where $\varphi$ is a homomorphism from $G$ into $G L_{m}(\mathbb{C}) ; m$ is a positive integer and $\varphi_{g}:=\varphi(g)$. A character is a class(conjugacy class) function. Many authors use a character to define a generalized matrix function, but, in this paper, we require such function to be a complex valued function because our main result hold whether the function is a character or not. However, we also investigate some interesting consequences when using a character.

Let $G \leq S_{n}$ and $\chi: G \rightarrow \mathbb{C}$ be a complex valued function. For each $A \in M_{n}(\mathbb{C})$, denote $[A]_{i j}$ the entry in the $i$-th row and $j$-th column of $A$. A function $d_{\chi}^{G}: M_{n}(\mathbb{C}) \rightarrow \mathbb{C}$ defined by

$$
d_{\chi}^{G}(A)=\sum_{\sigma \in G} \chi(\sigma) \prod_{i=1}^{n}[A]_{i \sigma(i)}
$$

is called a generalized matrix function associated with $G$ and $\chi$. We can see that $d_{\varepsilon}^{S_{n}}$ and $d_{1}^{S_{n}}$ are a determinant function and a permanent function, where $\varepsilon$ and 1 are the principal character and the alternating character, respectively. Moreover, for each $A \in M_{n}(\mathbb{C})$,

$$
d_{\chi}^{G}(A)=\sum_{\sigma \in S_{n}} \hat{\chi}(\sigma) \prod_{i=1}^{n}[A]_{i \sigma(i)},
$$


where $\hat{\chi}$ is an extension of $\chi$ which vanishes outside $G$. In this paper, we always consider $d_{\chi}^{G}$ as in the form (2.1).

A complex square matrix $A$ is called a symmetric matrix if $A^{T}=A$. We denote the set of all $n \times n$ complex symmetric matrices by $\mathbb{S}_{n}(\mathbb{C})$. For each $A, B \in \mathbb{S}_{n}(\mathbb{C})$, we have $(A B)^{T}=B A$.

\section{Main results}

We know that every permutation in $S_{n}$ can be uniquely written as a product of some disjoint cycles. Through out this paper, for every permutation $\sigma$ in $S_{n}$, the decomposition of $\sigma$ means the expression of $\sigma$ in term of a product of disjoint cycles not including a cycle of length 1 . For arbitrary positive integer $m$, the set $\{1, \ldots, m\}$ is symbolized by $[m]$. For each $\sigma \in S_{n}$, we denote $\operatorname{Fix}(\sigma):=\{i \in[n] \mid \sigma(i)=i\}$. For every cycle $\omega=\left(a_{1} \ldots a_{s}\right)$, we can see directly that $\left\{a_{1}, \ldots, a_{s}\right\}=\operatorname{Fix}(\omega)^{c}$, the complement of $\operatorname{Fix}(\omega)$. It is also clear that

" $\omega$ and $\pi$ are disjoint cycles if and only if $\operatorname{Fix}(\omega)^{c} \cap \operatorname{Fix}(\pi)^{c}=\emptyset "$.

Furthermore,

$$
\text { "Fix }(\omega \pi)^{c}=\operatorname{Fix}(\omega)^{c} \cup \operatorname{Fix}(\pi)^{c} \text { if } \omega \text { and } \pi \text { are disjoint cycles". }
$$

By using these facts, the following lemma holds.

Lemma 3.1. Let $\sigma \in S_{n}$. Suppose that $\sigma=\sigma_{1} \cdots \sigma_{k}$ is the decomposition of $\sigma$ and $i \in[n]$. Then the following statements hold.

(1) $i \in \operatorname{Fix}(\sigma)$ if and only if $i \in \operatorname{Fix}\left(\sigma_{j}\right)$ for every $j \in[k]$.

(2) If $i \in \operatorname{Fix}(\sigma)^{c}$, then there exists uniquely element $j \in[k]$ such that $i \in \operatorname{Fix}\left(\sigma_{j}\right)^{c}$.

(3) $\operatorname{Fix}(\sigma)^{c}=\operatorname{Fix}\left(\sigma_{1}\right)^{c} \cup \cdots \cup \operatorname{Fix}\left(\sigma_{k}\right)^{c}$.

If $i \in \operatorname{Fix}\left(\sigma_{j}\right)^{c}$, then, by (1), $i \in \operatorname{Fix}(\sigma)^{c}$. Moreover, because of (2), $i \in \operatorname{Fix}\left(\sigma_{l}\right)$ if $l \neq j$. So, $\sigma(i)=\sigma_{j}(i)$ if $i \in \operatorname{Fix}\left(\sigma_{j}\right)^{c}$. Note that $i \in$ $\operatorname{Fix}\left(\sigma_{j}\right)^{c}$ implies $\sigma_{j}^{t}(i) \in \operatorname{Fix}\left(\sigma_{j}\right)^{c}$ for every natural number $t$. Thus, by using mathematical induction,

$$
" \sigma^{t}(i)=\sigma_{j}^{t}(i) \text { if } i \in \operatorname{Fix}\left(\sigma_{j}\right)^{c} " .
$$


Now, for the decomposition $\sigma=\sigma_{1} \sigma_{2} \cdots \sigma_{k}$, we define the set $[\sigma]$ as follow.

$$
[\sigma]:=\left\{\sigma_{1}^{n_{1}} \sigma_{2}^{n_{2}} \cdots \sigma_{k}^{n_{k}} \mid n_{1}, \ldots, n_{k} \in\{1,-1\}\right\} .
$$

Define a relation $\sim$ on $S_{n}$ by, for each $\sigma, \tau \in S_{n}, \tau \sim \sigma$ if and only if $\tau \in[\sigma]$. We can see that, by the definition of $[\sigma], \sim$ is an equivalence relation and $[\sigma]$ is the equivalence class of $\sigma$.

Let $\omega=\left(a_{1} a_{2} \ldots a_{s}\right)$ be a cycle in $S_{n}$. If $s$ is even, we construct

$\omega_{\left(a_{1}\right)}:=\left(a_{1} a_{2}\right)\left(a_{3} a_{4}\right) \cdots\left(a_{s-1} a_{s}\right)$ and $\omega_{\left(a_{s}\right)}:=\left(a_{s} a_{1}\right)\left(a_{2} a_{3}\right) \cdots\left(a_{s-2} a_{s-1}\right)$,

and then denote $S(\omega):=\left\{\omega_{\left(a_{1}\right)}, \omega_{\left(a_{s}\right)}\right\}$. Define the set $X_{\omega}$ by

$$
X_{\omega}:= \begin{cases}\left\{\omega, \omega^{-1}\right\}, & \text { if } \omega \text { is a cycle of odd length } \\ \left\{\omega, \omega^{-1}\right\} \cup S(\omega), & \text { if } \omega \text { is a cycle of even length. }\end{cases}
$$

Note that, if $\omega$ is a transposition, then $X_{\omega}=\{\omega\}$. For every two subsets $A, B$ of $S_{n}$, denote $A B=\{\sigma \tau \mid \sigma \in A$ and $\tau \in B\}$. For any $\sigma \in S_{n}$, we define

$$
X_{\sigma}:=X_{\sigma_{1}} \cdots X_{\sigma_{k}}
$$

where $\sigma=\sigma_{1} \cdots \sigma_{k}$ is the decomposition of $\sigma$. By the definition of $[\sigma]$, we obtain that $[\sigma] \subseteq X_{\sigma}$. So, we can classify elements in $S_{n}$ into two types.

Definition 3.2. The permutation $\sigma$ is said to be type- $I$ if $X_{\sigma}=[\sigma]$ and it is said to be type-II if it is not a type-I.

Then every cycle of odd length is type-I. If $\omega$ is a cycle of even length, it is clear by the definition of $[\omega]$ and $X_{\omega}$ that $\omega$ is type-I if and only if it is a transposition. By the direct computation, $S(\omega)=S\left(\omega^{-1}\right)$, for each cycle $\omega$ of even length, and hence, $X_{\pi}=X_{\pi^{-1}}$, for every cycle $\pi$ in $S_{n}$. This implies that $X_{\sigma}=X_{\tau}$, for each $\tau \in[\sigma]$.

Proposition 3.3. Let $\sigma \in S_{n}$. Then $\sigma$ is type-II if and only if the decomposition of $\sigma$ contains a type-II cycle.

Proof. Let $\sigma=\sigma_{1} \cdots \sigma_{k}$ be the decomposition of $\sigma$. Assume that the decomposition does not contain any type-II cycle. Then $\left[\sigma_{i}\right]=\left\{\sigma_{i}, \sigma_{i}^{-1}\right\}=X_{\sigma_{i}}$ for every $1 \leq i \leq k$. So, by the definition of $[\sigma]$,

$$
X_{\sigma}=X_{\sigma_{1}} \cdots X_{\sigma_{k}}=\left[\sigma_{1}\right] \cdots\left[\sigma_{k}\right]=[\sigma] \text {, }
$$


that is, $\sigma$ is type-I. Conversely, suppose that the decomposition contains a type-II cycle, says $\sigma_{1}$. By considering $\sigma_{1}$ as $\sigma_{1}=\left(a_{1} \ldots a_{s}\right)$, we have $s \geq 4$ and $\left(\sigma_{1}\right)_{\left(a_{1}\right)} \sigma_{2} \sigma_{3} \cdots \sigma_{k} \in X_{\sigma}$. Since $\left(\sigma_{1}\right)_{\left(a_{1}\right)}$ is not equal to $\sigma_{1}$ nor $\sigma_{1}^{-1}$ and because of the uniqueness of the decomposition of a permutation, $\left(\sigma_{1}\right)_{\left(a_{1}\right)} \sigma_{2} \sigma_{3} \cdots \sigma_{k} \notin[\sigma]$, that is, $X_{\sigma} \neq[\sigma]$. Thus $\sigma$ is type-II.

Proposition 3.4. Let $\sigma \in S_{n}$ and $\tau \in X_{\sigma}$. Then the following statements hold.

(1) $\operatorname{Fix}(\sigma)=\operatorname{Fix}(\tau)$.

(2) $X_{\tau} \subseteq X_{\sigma}$.

(3) $X_{\tau}=X_{\sigma}$ if and only if $\tau \in[\sigma]$.

Proof. (1) It is straightforward by the definition of $X_{\sigma}$ that $\operatorname{Fix}(\sigma)^{c}=\operatorname{Fix}(\tau)^{c}$. Hence $\operatorname{Fix}(\sigma)=\operatorname{Fix}(\tau)$.

(2) Let $\sigma_{1} \cdots \sigma_{k}$ be the decomposition of $\sigma$. Then $\tau=\tau_{1} \cdots \tau_{k}$, where $\tau_{i} \in X_{\sigma_{i}}$. If $\tau_{i}=\sigma_{i}$ or $\sigma_{i}^{-1}$, then $X_{\tau_{i}}=X_{\sigma_{i}}$. Suppose that $\sigma_{i}$ is type-II and $\tau_{i}$ is not $\sigma_{i}$ nor $\sigma_{i}^{-1}$. We may consider $\sigma_{i}$ in the form $\sigma_{i}=\left(a_{1} \ldots a_{s}\right)$. Then $\tau_{i}$ is either $\left(\sigma_{i}\right)_{\left(a_{1}\right)}$ or $\left(\sigma_{i}\right)_{\left(a_{s}\right)}$. Both cases imply that $X_{\tau_{i}}=\left\{\tau_{i}\right\} \subseteq X_{\sigma_{i}}$. Thus $X_{\tau}=X_{\tau_{1}} \cdots X_{\tau_{k}} \subseteq X_{\sigma_{1}} \cdots X_{\sigma_{k}}=X_{\sigma}$.

(3) If $\tau \in[\sigma]$, we can conclude that $X_{\sigma}=X_{\tau}$ as we discuss before announcing Proposition 3.3. On the other hand, suppose that $X_{\tau}=X_{\sigma}$ and $\tau \notin[\sigma]$. By the above argument, there exists $i \in[k]$ such that $\tau_{i}=\left(\sigma_{i}\right)_{\left(a_{1}\right)}$ or $\tau_{i}=\left(\sigma_{i}\right)_{\left(a_{s}\right)}$, which implies that $X_{\tau_{i}} \neq X_{\sigma_{i}}$. This means that $X_{\tau} \neq X_{\sigma}$ which is a contradiction. Therefore $\tau \in[\sigma]$.

Proposition 3.5. Let $\sigma \in S_{n}$. Then the following statements hold.

(1) $X_{\sigma}$ is a union of the equivalence classes of permutations in $S_{n}$.

(2) If $\sigma$ contains exactly $l$ cycles of type-II, then the decomposition of each element of $X_{\sigma}$ contains at most $l$ type-II cycles.

(3) If $\sigma$ contains exactly l cycles of type-II, then $[\sigma]$ is the set of all elements of $X_{\sigma}$ containing exactly $l$ type-II cycles.

Proof. If $\sigma$ is type-I, then $X_{\sigma}=[\sigma]$. Suppose that $\sigma$ is type-II. Then the decomposition of $\sigma$ contains a type-II cycle. Let $\sigma=\sigma_{1} \cdots \sigma_{l} \sigma_{l+1} \cdots \sigma_{k}$ be the decomposition of $\sigma$, where $\sigma_{1}, \ldots, \sigma_{l}$ are all type-II cycles contained in this decomposition. For each $j \in[l]$, define

$$
\operatorname{Seq}(j):=\left\{\left(x_{1}, \ldots, x_{j}\right) \in \mathbb{N}^{j} \mid x_{i} \leq l \text { and } x_{1}<x_{2}<\cdots<x_{j}\right\} .
$$


For each sequence $(S)=\left(x_{1}, \ldots, x_{j}\right) \in S e q(j)$, denote $S:=\left\{x_{1}, \ldots, x_{j}\right\}$ and

$$
X_{\sigma}^{S}:=\prod_{i \in S} S\left(\sigma_{i}\right) \prod_{i \notin S}\left[\sigma_{i}\right] .
$$

Straightforward by this definition, we have $X_{\sigma}^{S} \subset X_{\sigma}$. Note that, for each $1 \leq j \leq l$,

$$
S\left(\sigma_{j}\right)=\left\{\left(\sigma_{j}\right)_{\left(a_{1}^{j}\right)},\left(\sigma_{j}\right)_{\left(a_{s_{j}}^{j}\right.}\right\}=\left[\left(\sigma_{j}\right)_{\left(a_{1}^{j}\right)}\right] \cup\left[\left(\sigma_{j}\right)_{\left(a_{s_{j}}^{j}\right)}\right],
$$

where $\sigma_{j}=\left(a_{1}^{j} \ldots a_{s_{j}}^{j}\right)$. Let $\mathbb{I}=\left\{a_{1}^{x_{1}}, a_{s_{x_{1}}}^{x_{1}}\right\} \times \cdots \times\left\{a_{1}^{x_{j}}, a_{s_{x_{j}}}^{x_{j}}\right\}$. Then

$$
X_{\sigma}^{S}=\bigcup_{\left(S_{x_{1}}, \ldots, S_{x_{j}}\right) \in \mathbb{I}}\left(\prod_{i \in S}\left[\left(\sigma_{i}\right)_{\left(S_{i}\right)}\right] \prod_{i \notin S}\left[\sigma_{i}\right]\right)=\bigcup_{\left(S_{x_{1}}, \ldots, S_{x_{j}}\right) \in \mathbb{I}}\left[\prod_{i \in S}\left(\sigma_{i}\right)_{\left(S_{i}\right)} \prod_{i \notin S} \sigma_{i}\right] .
$$

Thus $X_{\sigma}^{S}$ is a union of some equivalence classes of $S_{n}$. So, it suffices to show that $X_{\sigma}=[\sigma] \cup\left(\bigcup_{j=1}^{l} \bigcup_{(S) \in S e q(j)} X_{\sigma}^{S}\right)$. It is clear that

$$
[\sigma] \cup\left(\bigcup_{j=1}^{l} \bigcup_{(S) \in S e q(j)} X_{\sigma}^{S}\right) \subseteq X_{\sigma} .
$$

Let $\tau=\tau_{1} \cdots \tau_{k} \in X_{\sigma}$, where $\tau_{i} \in X_{\sigma_{i}}$. Suppose that $\tau \notin[\sigma]$. By using the same argument as in the proof of Proposition 3.4 the decomposition of $\tau$ contains an element in $S\left(\sigma_{i}\right)$ for some $1 \leq i \leq l$. Without loss of generality, we may assume that the decomposition of $\tau$ contains an element in each $S\left(\sigma_{1}\right), \ldots, S\left(\sigma_{j}\right)$ but not contain element in $S\left(\sigma_{j+1}\right), S\left(\sigma_{j+2}\right), \ldots, S\left(\sigma_{l}\right)$. Then

$$
\tau \in \prod_{i=1}^{j} S\left(\sigma_{i}\right) \prod_{i=j+1}^{k}\left[\sigma_{i}\right]=X_{\sigma}^{S_{0}},
$$

where $\left(S_{0}\right)=(1,2, \ldots, j)$. This implies that each element in $X_{\sigma}$ is contained in $[\sigma]$ or $\left(\bigcup_{j=1}^{l} \bigcup_{(S) \in S e q(j)} X_{\sigma}^{S}\right)$. So,

$$
X_{\sigma}=[\sigma] \cup\left(\bigcup_{j=1}^{l} \bigcup_{(S) \in S e q(j)} X_{\sigma}^{S}\right) .
$$


This proves that $(1)$ holds. For each $i \in[l]$, each cycle in $\left[\sigma_{i}\right]$ is type-II while every element in $S\left(\sigma_{i}\right)$ is type-I. Since $\sigma_{i}$ is type-I if $i \geq l$, by considering the construction of $X_{\sigma}^{S}$, where $S \in S e q(j)$, we can see that the decomposition of each element in $X_{\sigma}^{S}$ contains exactly $l-j$ type-II cycles. Thus (2) and (3) hold.

Definition 3.6. For each $\sigma \in S_{n}$, we define the $n \times n$ matrix $S_{\sigma}$ as

$$
\left[S_{\sigma}\right]_{i j}= \begin{cases}1, & \text { if } \sigma(i)=j \text { or } \sigma^{-1}(i)=j, \\ 0, & \text { otherwise. }\end{cases}
$$

It is not difficult to see that $S_{\sigma}$ is a symmetric matrix and $S_{\sigma}=S_{\sigma^{-1}}$. Furthermore, $S_{\sigma}=P_{\sigma}$ if and only if $\sigma^{2}=i d$, where $P_{\sigma}$ is the permutation matrix corresponding to $\sigma$.

Theorem 3.7. Let $G \leq S_{n}$ and $\chi: G \rightarrow \mathbb{C}$ be a complex valued function. For each $\sigma \in S_{n}, d_{\chi}^{G}\left(S_{\sigma}\right)=\sum_{\tau \in X_{\sigma}} \hat{\chi}(\tau)$.

Proof. Let $\sigma \in S_{n}$. To prove that $d_{\chi}^{G}\left(S_{\sigma}\right)=\sum_{\tau \in X_{\sigma}} \hat{\chi}(\tau)$, it suffices to show that $\left[S_{\sigma}\right]_{i \tau(i)}=1$ for each $i \in[n]$ if and only if $\tau \in X_{\sigma}$. Suppose that $\tau \in X_{\sigma}$. To show that $\left[S_{\sigma}\right]_{i \tau(i)}=1$ for each $i \in[n]$, it is enough to show that, for every $i \in[n], \tau(i)=\sigma(i)$ or $\tau(i)=\sigma^{-1}(i)$. Let $i \in[n]$. Note that, by Proposition 3.4, $\tau \in X_{\sigma}$ implies $\operatorname{Fix}(\sigma)=\operatorname{Fix}(\tau)$. Thus, if $i \in \operatorname{Fix}(\sigma)$, then $\tau(i)=i=\sigma(i)$. Now, suppose that $i \in \operatorname{Fix}(\sigma)^{c}$. Then there exists a unique cycle in the decomposition of $\sigma$, says $\pi$, such that $\sigma(i)=\pi(i)$. Since $\tau \in X_{\sigma}$, there exists a permutation $\theta \in X_{\pi}$ such that $\theta$ is contained in the decomposition of $\tau$. If $\pi$ is a cycle of odd length, then $X_{\pi}=\left\{\pi, \pi^{-1}\right\}$, which implies that $\theta=\pi$ or $\pi^{-1}$. Since $i \in \operatorname{Fix}(\pi)^{c}$ and $\theta \in X_{\pi}, i \in \operatorname{Fix}(\theta)^{c}$. By Lemma 3.1 $(2), \tau(i)=\theta(i)$ which is $\pi(i)$ or $\pi^{-1}(i)$. In other words,

$$
\tau(i)=\sigma(i) \text { or } \tau(i)=\sigma^{-1}(i) .
$$

If $\pi$ is a cycle of even length, then $X_{\pi}=\left\{\pi, \pi^{-1}\right\} \cup S(\pi)$. By using the same argument as the above, the proof is done in case $\theta \in\left\{\pi, \pi^{-1}\right\}$. Suppose that $\theta \in S(\pi)$. Since $i \in \operatorname{Fix}(\pi)^{c}$, we can write $\pi$ in the form $\left(i \pi(i) \pi^{2}(i) \ldots \pi^{s-1}(i)\right)$, where $s$ is the length of $\pi$. Then $\theta=\pi_{(i)}$ or $\theta=\pi_{\left(\pi^{s-1}(i)\right)}$. Since $\tau(i)=\theta(i)$,

$$
\tau(i)=\pi(i)=\sigma(i) \text { or } \tau(i)=\pi^{s-1}(i)=\pi^{-1}(i)=\sigma^{-1}(i) .
$$

Thus $\left[S_{\sigma}\right]_{i \tau(i)}=1$ for each $i \in[n]$. 
Conversely, suppose that $\left[S_{\sigma}\right]_{i \tau(i)}=1$ for every $i \in[n]$. Thus, for each $i \in[n]$,

$$
\tau(i)=\sigma(i) \text { or } \tau(i)=\sigma^{-1}(i),
$$

which implies that $i \in \operatorname{Fix}(\tau)$ if and only if $i \in \operatorname{Fix}(\sigma)$. So we obtain that

$$
\operatorname{Fix}(\sigma)=\operatorname{Fix}(\tau)
$$

Let $\sigma=\sigma_{1} \cdots \sigma_{k}$ and $\tau=\tau_{1} \cdots \tau_{l}$ be the decompositions of $\sigma$ and $\tau$, respectively. First of all, we will show that, for each $j \in[k]$, there exists $\theta \in X_{\sigma_{j}}$ such that $\theta$ is a factor of $\tau$. It suffices to work with $\sigma_{1}$ because the product of disjoint cycles are commute. Let $a \in \operatorname{Fix}\left(\sigma_{1}\right)^{c}$. Clearly, $a \notin \operatorname{Fix}(\sigma)=\operatorname{Fix}(\tau)$. There exists a unique cycle in the decomposition of $\tau$, without loss of generality, we may assume that it is $\tau_{1}$, such that $\tau_{1}(a)=\tau(a)$. If $\sigma_{1}$ is a transposition, then

$$
\sigma(a)=\sigma_{1}(a)=\sigma_{1}^{-1}(a)=\sigma^{-1}(a) .
$$

By applying (3.3), this condition implies that

$$
\tau_{1}(a)=\tau(a)=\sigma(a)=\sigma_{1}(a) \text { and } \tau_{1}^{2}(a)=\tau^{2}(a)=\tau \sigma(a) .
$$

By (3.3) again, $\tau \sigma(a)=\sigma^{-1} \sigma(a)$ or $\tau \sigma(a)=\sigma^{2}(a)$. Since $\sigma^{-1} \sigma(a)=a=$ $\sigma_{1}^{2}(a)=\sigma^{2}(a), \tau_{1}^{2}(a)=a$. Thus $\tau_{1}=\sigma_{1}$ if $\sigma_{1}$ is a transposition. This case is done by choosing $\theta=\tau_{1}$.

Assume that $\sigma_{1}$ is not a transposition. Without loss of generality, we can write

$$
\sigma_{1}=\left(a \sigma_{1}(a) \sigma_{1}^{2}(a) \ldots \sigma_{1}^{s-1}(a)\right) \text { and } \tau_{1}=\left(a \tau_{1}(a) \tau_{1}^{2}(a) \ldots \tau_{1}^{t-1}(a)\right) .
$$

Claim that, for each $x \in[t], \tau_{1}^{x}(a) \in \operatorname{Fix}\left(\sigma_{1}\right)^{c}$. To verify the claim, suppose that $x$ is the smallest element in $[t-1]$ such that $\tau_{1}^{x}(a) \in \operatorname{Fix}\left(\sigma_{1}\right)$. Since $\tau_{1}^{x}(a)=\tau_{1}\left(\tau_{1}^{x-1}(a)\right)=\tau\left(\tau_{1}^{x-1}(a)\right)$, by applying (3.3),

$$
\sigma_{1}\left(\tau_{1}^{x-1}(a)\right)=\sigma\left(\tau_{1}^{x-1}(a)\right)=\tau\left(\tau_{1}^{x-1}(a)\right)=\tau_{1}^{x}(a)
$$

or

$$
\sigma_{1}^{-1}\left(\tau_{1}^{x-1}(a)\right)=\sigma^{-1}\left(\tau_{1}^{x-1}(a)\right)=\tau\left(\tau_{1}^{x-1}(a)\right)=\tau_{1}^{x}(a) .
$$

If $\sigma_{1}\left(\tau_{1}^{x-1}(a)\right)=\tau_{1}^{x}(a)$, then

$$
\tau_{1}^{x-1}(a)=\sigma_{1}^{-1} \sigma_{1} \tau_{1}^{x-1}(a)=\sigma_{1}^{-1} \tau_{1}^{x}(a)=\tau_{1}^{x}(a),
$$


which is a contradiction. By the similar reasoning, the contradiction also occurs when $\sigma_{1}^{-1}\left(\tau_{1}^{x-1}(a)\right)=\tau_{1}\left(\tau_{1}^{x-1}(a)\right)=\tau_{1}^{x}(a)$ as we can see that

$$
\tau_{1}^{x-1}(a)=\sigma_{1} \sigma_{1}^{-1} \tau_{1}^{x-1}(a)=\sigma_{1} \tau_{1}^{x}(a)=\tau_{1}^{x}(a) .
$$

Thus, the claim is true. By applying the claim, we also have that $\operatorname{Fix}\left(\tau_{1}\right)^{c} \subseteq$ $\operatorname{Fix}\left(\sigma_{1}\right)^{c}$, so $s \geq t$. We now consider $\tau_{1}$ as four possible cases.

Case 1: $\tau_{1}$ is not a transposition and $\tau(a)=\sigma(a)$. Then $\tau_{1}(a)=\tau(a)=$ $\sigma(a)=\sigma_{1}(a)$. This indicates that $\tau_{1}^{2}(a)=\tau_{1} \sigma_{1}(a)$. If $\tau_{1} \sigma_{1}(a)=\tau \sigma_{1}(a)=$ $\sigma^{-1} \sigma_{1}(a)=\sigma_{1}^{-1} \sigma_{1}(a)=a$, then $\tau_{1}^{2}(a)=a$, which is not possible because $\tau_{1}$ is not a transposition. Then $\tau_{1}^{2}(a)=\sigma \sigma_{1}(a)=\sigma_{1}^{2}(a)$. By using the above arguments, we can show that

$$
\tau_{1}^{m}(a)=\sigma_{1}^{m}(a) \text {, for each } m \in[t-1] .
$$

Moreover, $\sigma_{1}^{t}(a)=\sigma \sigma_{1}^{t-1}(a)=\sigma \tau_{1}^{t-1}(a)$. If $\sigma \tau_{1}^{t-1}(a)=\tau^{-1} \tau_{1}^{t-1}(a)$, then $\sigma_{1}^{t}(a)=\tau_{1}^{t-2}(a)=\sigma_{1}^{t-2}(a)$, that is, $\sigma_{1}^{2}(a)=a$, which is a contradiction because $\sigma_{1}$ is not a transposition. Then

$$
\sigma_{1}^{t}(a)=\sigma \tau_{1}^{t-1}(a)=\tau \tau_{1}^{t-1}(a)=\tau_{1}^{t}(a)=a .
$$

So, we obtain that $s=t$ and hence $\tau_{1}=\sigma_{1}$. By setting $\theta=\tau_{1}$, this case is done.

Case 2: $\tau_{1}$ is not a transposition and $\tau(a)=\sigma^{-1}(a)$. By similar argument as Case 1 , we have $\tau_{1}=\sigma_{1}^{-1}$. Thus this case is proven by putting $\theta=\tau_{1}$.

Case 3: $\tau_{1}$ is a transposition and $\tau(a)=\sigma(a)$. Then $\tau_{1}(a)=\sigma_{1}(a)$ and $\tau_{1}^{2}(a)=a$. This implies that $\operatorname{Fix}\left(\tau_{1}\right)^{c}=\left\{a, \sigma_{1}(a)\right\}$. Since $\operatorname{Fix}(\tau)=\operatorname{Fix}(\sigma)$ and $\sigma_{1}^{2}(a) \in \operatorname{Fix}(\sigma)^{c}=\operatorname{Fix}(\tau)^{c}$, there exists a unique cycle in the decomposition of $\tau$, says $\tau_{2}$ such that $\sigma_{1}^{2}(a) \in \operatorname{Fix}\left(\tau_{2}\right)^{c}$. Because $\sigma_{1}$ is not a transposition, we have $\sigma_{1}^{2}(a) \neq a$. Since $\sigma_{1}^{2}(a) \notin \operatorname{Fix}\left(\tau_{1}\right)^{c}$, we can conclude that $\tau_{1} \neq \tau_{2}$, that is, $\tau_{1}$ and $\tau_{2}$ are disjoint. If $\tau \sigma_{1}^{2}(a)=\sigma^{-1} \sigma_{1}^{2}(a)$, then $\tau_{2} \sigma_{1}^{2}(a)=\sigma_{1}(a)=\tau_{1}(a)$, which is a contradiction because $\tau_{1}$ and $\tau_{2}$ are disjoint. This implies that $\tau_{2} \sigma_{1}^{2}(a)=\sigma \sigma_{1}^{2}(a)=\sigma_{1}^{3}(a)$. If $\tau_{2}$ is not a transposition, by using the same argument as case $1, \tau_{2}=\sigma_{1}$. This also contradicts to the fact that $\tau_{1}$ and $\tau_{2}$ are disjoint. Thus $\tau_{2}$ is a transposition. Note that $\sigma_{1}$ is not a transposition, that is, $s \geq 3$. If $s=3$, then $\tau_{1}=\left(a \sigma_{1}(a)\right)$ and $\tau_{2}=\left(\sigma_{1}^{2}(a) a\right)$, which is a contradiction, and hence $s \geq 4$. If $s=4$, this case is proved by choosing $\theta=\tau_{1} \tau_{2}=\left(\sigma_{1}\right)_{(a)}$. Suppose that $s>4$, recall the fact that $\operatorname{Fix}(\sigma)^{c}=\operatorname{Fix}(\tau)^{c}$ again, there exists a unique cycle in the decomposition of $\tau$, says $\tau_{3}$ such 
that $\sigma_{1}^{4}(a) \in \operatorname{Fix}\left(\tau_{3}\right)^{c}$. By using the same argument as above, we obtain the conclusion that $\tau_{3}$ is a transposition and $\tau_{3} \sigma_{1}^{4}(a)=\sigma_{1}^{5}(a)$. By repeating this method, we can conclude that, for each $m \in\left\{2, \ldots,\left\lfloor\frac{s}{2}\right\rfloor\right\}$,

$$
\tau_{m}=\left(\sigma_{1}^{2 m-2}(a) \sigma_{1}^{2 m-1}(a)\right)
$$

If $s$ is odd, then

$$
\tau_{\left\lfloor\frac{s}{2}\right\rfloor}=\left(\sigma_{1}^{s-1}(a) \sigma_{1}^{s}(a)\right)=\left(\sigma_{1}^{s-1}(a) a\right),
$$

which is a contradiction because $\tau_{1}$ and $\tau_{\left\lfloor\frac{s}{2}\right\rfloor}$ are disjoint. Thus $s$ is even. Moreover, $\tau_{1} \tau_{2} \cdots \tau_{\frac{s}{2}}=\left(\sigma_{1}\right)_{(a)}$. Choose $\theta=\left(\sigma_{1}\right)_{(a)}$. This case is verify.

Case 4: $\tau_{1}$ is a transposition and $\tau(a)=\sigma^{-1}(a)$. By using the similar reasoning as Case 3 and assigning $\theta=\left(\sigma_{1}\right)_{\left(\sigma_{1}^{s-1}(a)\right)}$, we obtain that $\theta$ is contained in the decomposition of $\tau$, and hence this case is done.

Now, we have the fact that, for every cycle $\pi$ in the decomposition of $\sigma$, there exists exactly one element $\theta \in X_{\pi}$ that contained in the decomposition of $\tau$. So, $\tau$ can be expressed as $\tau=\theta_{1} \cdots \theta_{q}$, where $q \geq k, \theta_{i} \in X_{\sigma_{i}}$, for each $i \in[k]$, and $\theta_{i}$ and $\theta_{j}$ are disjoint if $i \neq j$. If $q \neq k$, then

$$
\operatorname{Fix}(\tau)^{c}=\bigcup_{i=1}^{q} \operatorname{Fix}\left(\theta_{i}\right)^{c} \neq \bigcup_{i=1}^{k} \operatorname{Fix}\left(\theta_{i}\right)^{c}=\bigcup_{i=1}^{k} \operatorname{Fix}\left(\sigma_{i}\right)^{c}=\operatorname{Fix}(\sigma)^{c}
$$

which contradicts to (3.4). This implies that $q=k$ and hence $\tau \in X_{\sigma}$. Thus the statement of this theorem is proven.

Corollary 3.8. Let $\sigma \in S_{n}$. Then $\operatorname{perm}\left(S_{\sigma}\right)=\left|X_{\sigma}\right|$, where $\operatorname{perm}\left(S_{\sigma}\right)$ is the permanent of $S_{\sigma}$.

By using Theorem 3.7, we can find a necessary and sufficient condition for the equality of two generalized matrix functions on the set of all symmetric matrices as below.

Theorem 3.9. Let $H$ and $K$ be subgroups of $S_{n}$ and $\phi$ and $\psi$ complex valued functions of $H$ and $K$, respectively. Then the following conditions are equivalent.

(1) $d_{\phi}^{H}(A)=d_{\psi}^{K}(A)$ for each $A \in \mathbb{S}_{n}(\mathbb{C})$,

(2) $d_{\phi}^{H}\left(S_{\sigma}\right)=d_{\psi}^{K}\left(S_{\sigma}\right)$ for every $\sigma \in S_{n}$,

(3) $\sum_{\tau \in[\sigma]} \hat{\phi}(\tau)=\sum_{\tau \in[\sigma]} \hat{\psi}(\tau)$ for every $\sigma \in S_{n}$. 
Proof. It is obvious that (1) implies (2). Suppose that (2) is true. Let $\sigma=\sigma_{1} \cdots \sigma_{k}$ be a decomposition of $\sigma$. If $\sigma$ is type-I, i.e., $X_{\sigma}=[\sigma]$, then, by Theorem 3.7 ,

$$
\sum_{\tau \in[\sigma]} \hat{\phi}(\tau)=\sum_{\tau \in X_{\sigma}} \hat{\phi}(\tau)=d_{\phi}^{H}\left(S_{\sigma}\right)=d_{\psi}^{K}\left(S_{\sigma}\right)=\sum_{\tau \in X_{\sigma}} \hat{\psi}(\tau)=\sum_{\tau \in[\sigma]} \hat{\psi}(\tau) .
$$

Assume that $\sigma$ is type-II. By Proposition 3.3, the decomposition of $\sigma$ contains at least one type-II cycle. To show that $\sum_{\tau \in[\sigma]} \hat{\phi}(\tau)=\sum_{\tau \in[\sigma]} \hat{\psi}(\tau)$, for each $\sigma \in$ $S_{n}$, we will use a strong induction on the number of type-II cycle contained in each permutation. Firstly, suppose that $\sigma$ contains only one type-II cycle, without loss of generality, says $\sigma_{1}=\left(a_{1} \ldots a_{s}\right)$. By applying (3.2), we have

$$
X_{\sigma}=[\sigma] \cup S(\sigma)
$$

where $S(\sigma)=S\left(\sigma_{1}\right)\left[\sigma_{2}\right]\left[\sigma_{3}\right] \cdots\left[\sigma_{k}\right]$. By using Theorem 3.7, we obtain that

$$
\sum_{\tau \in X_{\sigma}} \hat{\phi}(\tau)=d_{\phi}^{H}\left(S_{\sigma}\right)=d_{\psi}^{K}\left(S_{\sigma}\right)=\sum_{\tau \in X_{\sigma}} \hat{\psi}(\tau)
$$

So, by Proposition 3.5, we can conclude that $[\sigma] \cap S(\sigma)=\emptyset$, and thus,

$$
\sum_{\tau \in[\sigma]} \hat{\phi}(\tau)+\sum_{\tau \in S(\sigma)} \hat{\phi}(\tau)=\sum_{\tau \in X_{\sigma}} \hat{\phi}(\tau)=\sum_{\tau \in X_{\sigma}} \hat{\psi}(\tau)=\sum_{\tau \in[\sigma]} \hat{\psi}(\tau)+\sum_{\tau \in S(\sigma)} \hat{\psi}(\tau) .
$$

Since each element in $S\left(\sigma_{1}\right)$ is type-I, every permutation in $S(\sigma)$ is also typeI. Moreover, by Theorem 3.7, $d_{\phi}^{H}\left(S_{\tau}\right)=\hat{\phi}(\tau)$ and $d_{\psi}^{K}\left(S_{\tau}\right)=\hat{\psi}(\tau)$, for each $\tau \in S(\sigma)$. As we have already proven that this theorem holds for every type-I permutation, we have

$$
\sum_{\tau \in S(\sigma)} \hat{\phi}(\tau)=\sum_{\tau \in S(\sigma)} d_{\phi}^{H}\left(S_{\tau}\right)=\sum_{\tau \in S(\sigma)} d_{\psi}^{K}\left(S_{\tau}\right)=\sum_{\tau \in S(\sigma)} \hat{\psi}(\tau) .
$$

This implies that

$$
\sum_{\tau \in[\sigma]} \hat{\phi}(\tau)=\sum_{\tau \in[\sigma]} \hat{\psi}(\tau)
$$

Because $\sigma$ is arbitrary, the statement of this theorem is true for every permutation containing exactly one type-II cycle in its decomposition. Suppose the hypothesis induction, that is, the decomposition of $\sigma$ contains exactly $l$ 
type-II cycles and the statement holds for each permutation containing less than $l$ type-II cycles. By (3.2),

$$
X_{\sigma}=[\sigma] \cup X
$$

where $X=\left(\bigcup_{j=1}^{l} \bigcup_{(S) \in S e q(j)} X_{\sigma}^{S}\right)$. By applying Proposition 3.5 and Theorem 3.7 again, we have $[\sigma] \cap X=\emptyset$ and

$$
\sum_{\tau \in[\sigma]} \hat{\phi}(\tau)+\sum_{\tau \in X} \hat{\phi}(\tau)=\sum_{\tau \in X_{\sigma}} \hat{\phi}(\tau)=\sum_{\tau \in X_{\sigma}} \hat{\psi}(\tau)=\sum_{\tau \in[\sigma]} \hat{\psi}(\tau)+\sum_{\tau \in X} \hat{\psi}(\tau) .
$$

By Proposition $3.5(3),[\sigma]$ is the set of all permutations in $X_{\sigma}$ containing $l$ type-II cycles in its decomposition, that is, the decomposition of each permutation in $X$ containing less than $l$ type-II cycles. By Proposition 3.5, $X$ is the union of the equivalence classes of elements in $X_{\sigma} \backslash[\sigma]$. Assume that $\sigma_{j}=\left(a_{1}^{j} \ldots a_{s_{j}}^{j}\right)$ for every $i \in[l]$. For each $(S)=\left(x_{1}, \ldots, x_{j}\right)$, due to (3.1),

$$
X_{\sigma}^{S}=\bigcup_{\left(S_{x_{1}}, \ldots, S_{x_{j}}\right) \in \mathbb{I}}\left[\prod_{i \in S}\left(\sigma_{i}\right)_{\left(S_{i}\right)} \prod_{i \notin S} \sigma_{i}\right]
$$

where $\mathbb{I}=\left\{a_{1}^{x_{1}}, a_{s_{x_{1}}}^{x_{1}}\right\} \times \cdots \times\left\{a_{1}^{x_{j}}, a_{s_{x_{j}}}^{x_{j}}\right\}$. Since $\prod_{i \in S}\left(\sigma_{i}\right)_{\left(S_{i}\right)} \prod_{i \notin S} \sigma_{i} \in X$, its decomposition contains less than $l$ type-II cycles. Because of the hypothesis of the induction, we have

$$
\sum_{\tau \in\left[\prod_{i \in S}\left(\sigma_{i}\right)_{\left(S_{i}\right)}\right.} \hat{\phi \notin S S} \sigma_{i \notin j} \hat{\phi}(\tau)=\sum_{\tau \in\left[\prod_{i \in S}\left(\sigma_{i}\right)_{\left(S_{i}\right)}\right.} \hat{\left.\prod_{i \notin S} \sigma_{i}\right]} \hat{\psi}(\tau) .
$$

Thus, by the definition of $X$ and (3.5), we obtain that

$$
\sum_{\tau \in X} \hat{\phi}(\tau)=\sum_{\tau \in X} \hat{\psi}(\tau)
$$

This implies that

$$
\sum_{\tau \in[\sigma]} \hat{\phi}(\tau)=\sum_{\tau \in[\sigma]} \hat{\psi}(\tau)
$$

The induction is done, and hence the statement is true for every permutation in $S_{n}$. 
Now, suppose that (3) occurs. Let $A$ be a symmetric matrix, $\sigma=\sigma_{1} \cdots \sigma_{k}$ the decomposition of $\sigma$ and $\omega \in[\sigma]$. Then $\omega=\sigma_{1}^{n_{1}} \cdots \sigma_{k}^{n_{k}}$, where $n_{1}, \ldots, n_{k} \in$ $\{1,-1\}$. Claim that $\prod_{i=1}^{n}[A]_{i \sigma(i)}=\prod_{i=1}^{n}[A]_{i \omega(i)}$. In order to prove that $(1)$ holds, we consider $\sigma_{j}$, for each $1 \leq j \leq k$. If $n_{j}=1$, then $\sigma(i)=\sigma_{j}(i)=\omega(i)$ for each $i \in \operatorname{Fix}\left(\sigma_{j}\right)^{c}$, which implies that

$$
\prod_{i \in \operatorname{Fix}\left(\sigma_{j}\right)^{c}}[A]_{i \sigma(i)}=\prod_{i \in \operatorname{Fix}\left(\sigma_{j}\right)^{c}}[A]_{i \omega(i)} .
$$

Suppose that $n_{j}=-1$, that is, $\sigma^{-1}(i)=\sigma_{j}^{-1}(i)=\omega(i)$ for every $i \in \operatorname{Fix}\left(\sigma_{j}\right)^{c}$. We obtain that

$$
\begin{aligned}
\prod_{i \in \operatorname{Fix}\left(\sigma_{j}\right)^{c}}[A]_{i \sigma(i)} & =\prod_{i \in \operatorname{Fix}\left(\sigma_{j}\right)^{c}}[A]_{\sigma(i) i} \\
& =\prod_{i \in \operatorname{Fix}\left(\sigma_{j}\right)^{c}}[A]_{i \sigma^{-1}(i)} \\
& =\prod_{i \in \operatorname{Fix}\left(\sigma_{j}\right)^{c}}[A]_{i \omega(i)} .
\end{aligned}
$$

By Lemma 3.1(3),

$$
[n]=\operatorname{Fix}(\sigma) \cup \operatorname{Fix}\left(\sigma_{1}\right)^{c} \cup \operatorname{Fix}\left(\sigma_{2}\right)^{c} \cup \cdots \cup \operatorname{Fix}\left(\sigma_{k}\right)^{c} .
$$

By recognizing that $\operatorname{Fix}(\sigma)=\operatorname{Fix}(\omega)$, we have

$$
\begin{aligned}
\prod_{i=1}^{n}[A]_{i \sigma(i)} & =\prod_{j=1}^{k} \prod_{i \in \operatorname{Fix}\left(\sigma_{j}\right)^{c}}[A]_{i \sigma(i)} \prod_{i \in \operatorname{Fix}(\sigma)}[A]_{i \sigma(i)} \\
& =\prod_{j=1}^{k} \prod_{i \in \operatorname{Fix}\left(\sigma_{j}\right)^{c}}[A]_{i \omega(i)} \prod_{i \in \operatorname{Fix}(\sigma)}[A]_{i \omega(i)} \\
& =\prod_{i=1}^{n}[A]_{i \omega(i)} .
\end{aligned}
$$

The claim is true. Now, let $\left[\tau_{1}\right], \ldots,\left[\tau_{s}\right]$ be all distinct equivalence classes of $S_{n}$. Then, by the fact above, we can conclude that

$$
d_{\phi}^{H}(A)=\sum_{\sigma \in S_{n}} \hat{\phi}(\sigma) \prod_{i=1}^{n}[A]_{i \sigma(i)}
$$




$$
\begin{aligned}
& =\sum_{j=1}^{k} \sum_{\sigma \in\left[\tau_{j}\right]} \hat{\phi}(\sigma) \prod_{i=1}^{n}[A]_{i \sigma(i)} \\
& =\sum_{j=1}^{k} \sum_{\sigma \in\left[\tau_{j}\right]} \hat{\phi}(\sigma) \prod_{i=1}^{n}[A]_{i \tau_{j}(i)} \\
& =\sum_{j=1}^{k}\left(\sum_{\sigma \in\left[\tau_{j}\right]} \hat{\phi}(\sigma)\right) \prod_{i=1}^{n}[A]_{i \tau_{j}(i)} \\
& =\sum_{j=1}^{k}\left(\sum_{\sigma \in\left[\tau_{j}\right]} \hat{\psi}(\sigma)\right) \prod_{i=1}^{n}[A]_{i \tau_{j}(i)} \\
& =\sum_{j=1}^{k} \sum_{\sigma \in\left[\tau_{j}\right]} \hat{\psi}(\sigma) \prod_{i=1}^{n}[A]_{i \tau_{j}(i)} \\
& =\sum_{j=1}^{k} \sum_{\sigma \in\left[\tau_{j}\right]} \hat{\psi}(\sigma) \prod_{i=1}^{n}[A]_{i \sigma(i)} \\
& =\sum_{\sigma \in S_{n}} \hat{\psi}(\sigma) \prod_{i=1}^{n}[A]_{i \sigma(i)} \\
& =d_{\psi}^{K}(A) .
\end{aligned}
$$

This proves (1).

Corollary 3.10. Let $H$ and $K$ be subgroups of $S_{n}$ and $\phi$ and $\psi$ complex valued functions of $H$ and $K$, respectively. Suppose that $Y$ is a subset of $\mathbb{S}_{n}(\mathbb{C})$ containing $S_{\sigma}$ for every $\sigma \in S_{n}$. Then $d_{\phi}^{H}(A)=d_{\psi}^{K}(A)$ for each $A \in Y$ if and only if $d_{\phi}^{H}\left(S_{\sigma}\right)=d_{\psi}^{K}\left(S_{\sigma}\right)$ for each $\sigma \in S_{n}$.

By Theorem 2.2. in [3], any two generalized matrix functions $d_{\phi}^{H}$ and $d_{\psi}^{K}$ are equal on the set of all nonsingular matrices if and only if $\hat{\phi}=\hat{\psi}$. Next corollary is obtained immediately by using this fact.

Corollary 3.11. Let $H$ and $K$ be subgroups of $S_{n}$. Suppose that $\phi$ and $\psi$ be complex valued functions of $H$ and $K$, respectively. If $d_{\phi}^{H}(A)=d_{\psi}^{K}(A)$ for every nonsingular matrix $A$, then $d_{\phi}^{H}(A)=d_{\psi}^{K}(A)$ for every $A \in \mathbb{S}_{n}(\mathbb{C})$.

We set $H=S_{n}=K$. By applying Theorem 3.9 , the results when $\phi$ and $\psi$ are characters are verified. 
Corollary 3.12. Let $\phi$ and $\psi$ be characters of $S_{n}$. Then $d_{\phi}^{S_{n}}(A)=d_{\psi}^{S_{n}}(A)$ for every $A \in \mathbb{S}_{n}(\mathbb{C})$ if and only if $\phi=\psi$.

Proof. Consider the equivalence class in $S_{n}$. We can see that each equivalence class is a subset of a conjugacy class. Since any character is a class function, for each $\sigma \in S_{n}$, we have $\phi(\tau)=\phi(\sigma)$ and $\psi(\tau)=\psi(\sigma)$ for every $\tau \in[\sigma]$. By Theorem [3.9, this corollary holds.

Theorem 3.13. Let $G \leq S_{n}$ and $\chi$ be a complex valued function of $G$. Then the following are equivalent.

(1) $d_{\chi}^{G}(A)=d_{\chi}^{G}\left(A^{T}\right)$ for each $A \in M_{n}(\mathbb{C})$,

(2) $d_{\chi}^{G}(A B)=d_{\chi}^{G}(B A)$ for each $A, B \in \mathbb{S}_{n}(\mathbb{C})$,

(3) $\chi(\sigma)=\chi\left(\sigma^{-1}\right)$ for each $\sigma \in S_{n}$.

Proof. Since $(A B)^{T}=B A$ for every symmetric matrices $A$ and $B$, (1) implies (2). Suppose that (2) is true. Let $\sigma \in S_{n}$. There exist symmetric matrices $A$ and $B$ such that $P_{\sigma}=A B$, where $P_{\sigma}$ is the permutation matrix corresponding to $\sigma$. So $P_{\sigma^{-1}}=P_{\sigma}^{T}=B A$. By the assertion,

$$
\chi(\sigma)=d_{\chi}^{G}\left(P_{\sigma}\right)=d_{\chi}^{G}\left(P_{\sigma^{-1}}\right)=\chi\left(\sigma^{-1}\right) .
$$

Suppose that (3) holds, that is, $\chi(\sigma)=\chi\left(\sigma^{-1}\right)$ for every $\sigma \in S_{n}$. Let $A \in M_{n}(\mathbb{C})$. Then

$$
\begin{aligned}
d_{\chi}^{G}(A) & =\sum_{\sigma \in G} \chi(\sigma) \prod_{i=1}^{n}[A]_{i \sigma(i)} \\
& =\sum_{\sigma \in G} \chi(\sigma) \prod_{i=1}^{n}[A]_{\sigma^{-1}(i) i} \\
& =\sum_{\sigma \in G} \chi\left(\sigma^{-1}\right) \prod_{i=1}^{n}[A]_{\sigma(i) i} \\
& =\sum_{\sigma \in G} \chi(\sigma) \prod_{i=1}^{n}[A]_{\sigma(i) i} \\
& =d_{\chi}^{G}\left(A^{T}\right),
\end{aligned}
$$

which completes the proof.

Since a character is a class(conjugacy class) function, next corollaries are obtained immediately. 
Corollary 3.14. Let $G \leq S_{n}$ and $\chi$ be a character of $G$. Then the following are equivalent.

(1) $d_{\chi}^{G}(A)=d_{\chi}^{G}\left(A^{T}\right)$ for each $A \in M_{n}(\mathbb{C})$,

(2) $d_{\chi}^{G}(A B)=d_{\chi}^{G}(B A)$ for each $A, B \in \mathbb{S}_{n}(\mathbb{C})$,

(3) $\chi$ is a real valued function.

Corollary 3.15. Let $\chi$ be a character of $S_{n}$. Then $d_{\chi}^{S_{n}}(A B)=d_{\chi}^{S_{n}}(B A)$ for every $A, B \in \mathbb{S}_{n}(\mathbb{C})$.

For each $\sigma \in S_{n}$, define $C_{\sigma}:[n] \times[n] \rightarrow\{0,1\}$ by

$$
C_{\sigma}(i, j)= \begin{cases}1, & \text { if } i, j \in \operatorname{Fix}(\sigma)^{c} \\ 0, & \text { otherwise. }\end{cases}
$$

Lemma 3.16. Let $\sigma=\left(a_{1} a_{2} a_{3}\right) \in S_{n}$. Then

$$
\left[S_{\sigma}^{2}\right]_{i j}=C_{\sigma}(i, j)+\delta_{i j}
$$

where $\delta_{i j}$ is the Kronecker delta function.

Proof. By the definition of $S_{\sigma}$, we have $\left[S_{\sigma}\right]_{i j} \in\{0,1\}$ and $\left[S_{\sigma}\right]_{i j}=1$ if and only if $j=\sigma(i)$ or $j=\sigma^{-1}(i)$ for each $i, j \in[n]$. Note that

$$
\left[S_{\sigma}^{2}\right]_{i j}=\sum_{k=1}^{n}\left[S_{\sigma}\right]_{i k}\left[S_{\sigma}\right]_{k j} .
$$

We consider $\left[S_{\sigma}\right]_{i j}$, where $i, j \in[n]$, as six possible cases. By applying the equation (3.6) in each case, we can verify this lemma as below.

Case 1. $i, j \in \operatorname{Fix}(\sigma)$ and $i=j$. Then $C_{\sigma}(i, j)=0$ and hence

$$
\left[S_{\sigma}^{2}\right]_{i j}=\left[S_{\sigma}^{2}\right]_{i i}=\left(\left[S_{\sigma}\right]_{i i}\right)^{2}=1=C_{\sigma}(i, j)+\delta_{i j} .
$$

Case 2. $i, j \in \operatorname{Fix}(\sigma)$ but $i \neq j$. So, we obtain that $\left[S_{\sigma}\right]_{i k}=0$ for each $k \in\{1 \ldots, n\} \backslash\{i\}$, which implies that $\left[S_{\sigma}\right]_{i j}=0$. Thus

$$
\left[S_{\sigma}^{2}\right]_{i j}=\left[S_{\sigma}\right]_{i i}\left[S_{\sigma}\right]_{i j}=0=C_{\sigma}(i, j)+\delta_{i j} .
$$

Case 3. $i, j \in \operatorname{Fix}(\sigma)^{c}$ and $i=j$. Then $\left[S_{\sigma}\right]_{i k}=1$ as long as $k$ is $\sigma(i)$ or $\sigma^{-1}(i)$. This indicates that

$$
\left[S_{\sigma}^{2}\right]_{i j}=\left[S_{\sigma}\right]_{i \sigma(i)}\left[S_{\sigma}\right]_{\sigma(i) j}+\left[S_{\sigma}\right]_{i \sigma^{-1}(i)}\left[S_{\sigma}\right]_{\sigma^{-1}(i) j}
$$




$$
\begin{aligned}
& =\left[S_{\sigma}\right]_{i \sigma(i)}\left[S_{\sigma}\right]_{\sigma(i) i}+\left[S_{\sigma}\right]_{i \sigma^{-1}(i)}\left[S_{\sigma}\right]_{\sigma^{-1}(i) i} \\
& =2 \\
& =C_{\sigma}(i, j)+\delta_{i j} .
\end{aligned}
$$

Case 4. $i, j \in \operatorname{Fix}(\sigma)^{c}$ but $i \neq j$. By a similar argument as above,

$$
\left[S_{\sigma}^{2}\right]_{i j}=\left[S_{\sigma}\right]_{i \sigma(i)}\left[S_{\sigma}\right]_{\sigma(i) j}+\left[S_{\sigma}\right]_{i \sigma^{-1}(i)}\left[S_{\sigma}\right]_{\sigma^{-1}(i) j} .
$$

Since $|\operatorname{Fix}(\sigma)|=3$ and $i \neq j$, either $j=\sigma(i)$ or $j=\sigma^{-1}(i)$, which implies

$$
\left[S_{\sigma}^{2}\right]_{i j}=1=C_{\sigma}(i, j)+\delta_{i j}
$$

Case 5. $i \in \operatorname{Fix}(\sigma)$ and $j \in \operatorname{Fix}(\sigma)^{c}$. Then $j \neq \sigma(i)$, that is, $\left[S_{\sigma}\right]_{i j}=0$. Thus

$$
\left[S_{\sigma}^{2}\right]_{i j}=\left[S_{\sigma}\right]_{i i}\left[S_{\sigma}\right]_{i j}=0=C_{\sigma}(i, j)+\delta_{i j} .
$$

Case 6. $i \in \operatorname{Fix}(\sigma)^{c}$ and $j \in \operatorname{Fix}(\sigma)$. By using a similar reasoning as Case 5.

$$
\left[S_{\sigma}^{2}\right]_{i j}=\left[S_{\sigma}\right]_{i j}\left[S_{\sigma}\right]_{j j}=0=C_{\sigma}(i, j)+\delta_{i j} .
$$

Thus the proof is completed.

Denote $F_{3}^{c}(n):=\left\{\sigma \in S_{n}|| \operatorname{Fix}(\sigma)^{c} \mid \leq 3\right\}$. By using Lemma 3.16, the following theorem is obtained.

Theorem 3.17. Let $G$ be a subgroup of $S_{n}$ and $\chi$ a character of $G$. Then $d_{\chi}^{G}=\operatorname{det}$ if and only if $d_{\chi}^{G}\left(S_{\sigma}\right) d_{\chi}^{G}\left(S_{\tau}\right)=d_{\chi}^{G}\left(S_{\sigma} S_{\tau}\right)$, for every $\sigma, \tau \in F_{3}^{c}(n)$.

Proof. Let $\sigma, \tau \in F_{3}^{c}(n)$. Clearly, $d_{\chi}^{G}\left(S_{\sigma}\right) d_{\chi}^{G}\left(S_{\tau}\right)=d_{\chi}^{G}\left(S_{\sigma} S_{\tau}\right)$ if $d_{\chi}^{G}=\operatorname{det}$. Suppose that the converse hypothesis is true. Since $S_{i d}=I$ and $\chi(i d)=$ $\operatorname{det}\left(S_{i d}\right)$, by the assumption, we have

$$
\chi(i d)=d_{\chi}^{G}\left(S_{i d}\right)=\left(d_{\chi}^{G}\left(S_{i d}\right)\right)^{2}=(\chi(i d))^{2} .
$$

Because $\chi$ is a character, $\chi(i d) \neq 0$, that is, $\chi(i d)=1$. Thus $\chi$ is linear. If $n=1$, then $d_{\chi}^{G}(A)=[A]_{11}=\operatorname{det}(A)$ for arbitrary $A \in M_{n}(\mathbb{C})$. For each transposition $\omega$ in $S_{n}$, we have $\omega^{2}=i d$, so, by the assumption,

$$
(\hat{\chi}(\omega))^{2}=\left(d_{\chi}^{G}\left(S_{\omega}\right)\right)^{2}=d_{\chi}^{G}(I)=1 .
$$


This implies that $\hat{\chi}(\omega)$ is either 1 or -1 , that is, $\omega \in G$. If $n=2$ and $\chi(12)=1$, then

$$
128=d_{\chi}^{G}\left(\left[\begin{array}{ll}
8 & 8 \\
8 & 8
\end{array}\right]\right)=d_{\chi}^{G}\left(\left[\begin{array}{ll}
2 & 2 \\
2 & 2
\end{array}\right]\right) d_{\chi}^{G}\left(\left[\begin{array}{ll}
2 & 2 \\
2 & 2
\end{array}\right]\right)=64
$$

which is a contradiction. This implies that $\chi(12)=-1$, and thus $d_{\chi}^{G}=\operatorname{det}$. Suppose that $n \geq 3$. Since the set of all transpositions is the generating set of $S_{n}, G=S_{n}$. For each $\sigma \in S_{n}$, we can write

$$
\sigma=\theta_{1} \theta_{2} \cdots \theta_{k}
$$

where $\theta_{m}$ is a transposition for each $m \in[k]$. Because $\chi$ is linear, we can conclude that

$$
\chi(\sigma)=\chi\left(\theta_{1}\right) \chi\left(\theta_{2}\right) \cdots \chi\left(\theta_{k}\right) .
$$

Thus, to verify that $d_{\chi}^{G}=$ det, it suffices to show that $\chi(\omega)=-1$ for each transposition $\omega$. Let $\sigma=\left(a_{1} a_{2} a_{3}\right)$. By Theorem 3.7 and the assumption,

$$
d_{\chi}^{G}\left(S_{\sigma}^{2}\right)=\left(d_{\chi}^{G}\left(S_{\sigma}\right)\right)^{2}=\left(\chi\left(a_{1} a_{2} a_{3}\right)+\chi\left(a_{1} a_{3} a_{2}\right)\right)^{2} .
$$

By Lemma [3.16, we have $\left[S_{\sigma}^{2}\right]_{i j}=0$ if and only if $i \neq j$ and at least one of $i, j$ is in $\operatorname{Fix}(\sigma)$ and the other is in $\operatorname{Fix}(\sigma)^{c}$. Thus, for each $\tau \in B:=$ $\left\{i d,\left(a_{1} a_{2}\right),\left(a_{1} a_{3}\right),\left(a_{2} a_{3}\right), \sigma, \sigma^{-1}\right\}, \prod_{i=1}^{n}\left[S_{\sigma}^{2}\right]_{i \tau(i)} \neq 0$ while $\prod_{i=1}^{n}\left[S_{\sigma}^{2}\right]_{i \pi(i)}=0$ for every $\pi \in S_{n} \backslash B$. Therefore,

$$
\begin{aligned}
d_{\chi}^{G}\left(S_{\sigma}^{2}\right) & =\sum_{\tau \in S_{n}} \chi(\tau) \prod_{i=1}^{n}\left[S_{\sigma}^{2}\right]_{i \tau(i)} \\
& =\sum_{\tau \in B} \chi(\tau) \prod_{i=1}^{n}\left(C_{\sigma}(i, \tau(i))+\delta_{i \tau(i)}\right) \\
& =\chi\left(\sigma^{-1}\right)+\chi(\sigma)+2 \chi\left(a_{1} a_{2}\right)+2 \chi\left(a_{1} a_{3}\right)+2 \chi\left(a_{2} a_{3}\right)+8 \chi(i d) .
\end{aligned}
$$

Note that

$$
\sigma=\left(a_{1} a_{3}\right)\left(a_{1} a_{2}\right)=\left(a_{1} a_{2}\right)\left(a_{2} a_{3}\right)=\left(a_{2} a_{3}\right)\left(a_{1} a_{3}\right)
$$

and

$$
\sigma^{-1}=\left(a_{1} a_{2}\right)\left(a_{1} a_{3}\right)=\left(a_{2} a_{3}\right)\left(a_{1} a_{2}\right)=\left(a_{1} a_{3}\right)\left(a_{2} a_{3}\right) .
$$


Since $\chi$ is linear, we can compute that

$$
\chi\left(a_{1} a_{2}\right)=\chi\left(a_{1} a_{3}\right)=\chi\left(a_{2} a_{3}\right) \text { and } \chi(\sigma)=\left(\chi\left(a_{1} a_{2}\right)\right)^{2}=\chi\left(\sigma^{-1}\right) .
$$

By applying (3.8), we have $\chi(\sigma)=1$, which implies that

$$
d_{\chi}^{G}\left(S_{\sigma}^{2}\right)=6 \chi\left(a_{1} a_{2}\right)+10 .
$$

Similarly, by applying (3.8) to (3.7), we obtain that

$$
d_{\chi}^{G}\left(S_{\sigma}^{2}\right)=4
$$

Consider (3.9) and (3.10), we have $\chi\left(a_{1} a_{2}\right)=-1$. Since $a_{1}, a_{2}$ are arbitrary, $\chi(\omega)=-1$ for every transposition $\omega$ in $S_{n}$. Hence $d_{\chi}^{G}=$ det.

Due to Theorem 3.17, when we define a generalized matrix function by using a character of a subgroup of $S_{n}$, det is the unique generalized matrix function preserving the product on every subset of $M_{n}(\mathbb{C})$ containing $F_{3}^{c}(n)$. An obvious example of such subset is $\mathbb{S}_{n}(\mathbb{C})$. Moreover, by Theorem 3.7, $S_{\sigma}$ is nonsingular for every $\sigma \in F_{3}^{c}(n)$. So, $G L_{n}(\mathbb{C})$ is also a subset of $M_{n}(\mathbb{C})$ containing $F_{3}^{c}(n)$.

\section{Acknowledgment}

The second author would like to thank Faculty of Sciences, Naresuan University, for the financial support on the project number R2561E003.

\section{References}

[1] A. J. Bosch, The factorization of a square matrix into two symmetric matrices, The American Mathmetical Monthly 93 (6) (1986) 462-464.

[2] M. H. Jafari, A. R. Madadi, Generalized matrix functions and determinants, Central European Journal of Mathematics 12 (3) (2014) 464-469.

[3] M. H. Jafari, A. R. Madadi, On the equality of generalized matrix functions, Linear Algebra and its Applications 456 (2014) 16-21. 